\title{
Synthetic cannabinoids induce acute lung inflammation via cannabinoid receptor 1 activation
}

\section{To the Editor:}

Synthetic cannabinoid (SC) use has persisted in the United States despite schedule-1 placement under the Synthetic Drug Abuse Prevention Act of 2012 [1]. Analysis of the National Poison Data System indicates that hospitalisations caused by SC use increased significantly between 2010 and 2015 [2]. Moreover, there is a trend of the increasing use of such compounds among adolescents [3]. SCs are often 30-100-fold more potent than $\Delta^{9}$-tetrahydrocannabinol (THC), the major psychoactive ingredient of cannabis, in activating Cannabinoid receptor $1\left(\mathrm{CB}_{1} \mathrm{R}\right)$. Users are attracted to SCs because of the cheaper, novel and stronger highs such substances offer compared to cannabis, and because the compounds are not screened for in typical drug tests $[1,2]$. Among those hospitalised for SC use, some patients exhibited respiratory failure [4-7], pulmonary infiltrates [5, 7], alveolar damage or haemorrhage [5-7] and histopathologic features similar to organising pneumonia [4-6]. The mechanism by which SCs damage pulmonary tissue has yet to be elucidated - whether by $\mathrm{SC}$ binding at $\mathrm{CB}_{1} \mathrm{R}, \mathrm{CB}_{2} \mathrm{R}$ or another receptor, and what downstream effects such binding elicits. Solving this conundrum is the first step in optimising treatment for patients presenting with SC-related respiratory distress.

$\mathrm{CB}_{1} \mathrm{R}$ is expressed in multiple cell types in the lung, wherein its overstimulation and overactivity has been linked to lung injury, alveolar inflammation and fibrosis $[8,9]$. Here, we explored the acute effects of the SC $\mathrm{CP} 55,940$ on $\mathrm{CB}_{1} \mathrm{R}$ activation and inflammation in the lungs of C57BL6/J mice. CP55,940 is a functional and structural analogue of the compounds JWH-018 and CP47,497, which have been extensively used in SC "K2/Spice" blends. Animal procedures were conducted according to regulations of the Institutional Animal Care and Use Committee of the National Institute on Alcohol Abuse and Alcoholism. CP55,940 $\left(22.6 \mu \mathrm{g} \cdot \mathrm{kg}^{-1}, 565 \mathrm{ng}\right.$ in $\left.150 \mu \mathrm{L}\right)$ was delivered in vehicle (0.01\% DMSO, $0.5 \%$ Tween $80,99.49 \%$ saline) via oropharyngeal (OP) aspiration to mice of $25-27 \mathrm{~g}$ body weight. This dose was estimated to generate similar neurobiological and behavioural effects as experienced by a human smoking two cannabis cigarettes [10]. The lung tissue was collected 4,24 and $48 \mathrm{~h}$ after CP55,940 delivery to assess gene expression of cannabinoid receptors, inflammatory cytokines, chemokines and pro-inflammatory transcription factors using the method previously described [8]. Bioactive lipids were quantified by mass spectrometry.

OP CP55,940 administration significantly increased gene expression of $\mathrm{CB}_{1} \mathrm{R}$ and $\mathrm{CB}_{2} \mathrm{R}$ within $4 \mathrm{~h}$ with mRNA levels returning to normal by $48 \mathrm{~h}$ post-drug delivery (figure 1a). Additionally, CP55,940 strongly induced the expression of inflammatory cytokines, including tumour necrosis factor alpha (Tnfo), interleukin 1 beta (IL1 $\beta$ ) and interleukin 6 (IL6); chemokines, including chemokine (C-X-C motif) ligand 1 ( $\mathrm{Cxcl}$ ), chemokine (C-C motif) ligand $2(\mathrm{Ccl} 2)$, chemokine (C-C motif) ligand $3(\mathrm{Ccl} 3)$ and chemokine (C-X-C motif) ligand 10 (Cxcl10) (figure $1 \mathrm{~b})$; as well as proinflammatory transcription factors such as interferon regulatory factor 5 (Irf5) and NACHT, LRR and PYD domains-containing protein 3 (Nlrp3) inflammasome (figure $1 \mathrm{~b}$ ) with a similar time course. The wet weight of the lung was significantly increased at $4 \mathrm{~h}$ after CP55,940 administration (figure 1c), suggesting pulmonary oedema due to lung inflammation. Since the most significant gene expression changes were also observed at 4 -h time-point, we tried to block

$@$ ERSpublications

Synthetic cannabinoids (SCs) induce a pro-inflammatory condition by activating cannabinoid receptor $1\left(C_{1} R\right)$ in the lungs of mice, which raises a potential therapeutic use of $C_{1} R$ antagonists in SC-induced lung disease resulting in hospitalisation https://bit.ly/31bWw4Q

Cite this article as: Zawatsky CN, Abdalla J, Cinar R. Synthetic cannabinoids induce acute lung inflammation via cannabinoid receptor 1 activation. ERJ Open Res 2020; 6: 00121-2020 [https://doi.org/10.1183/23120541.00121-2020].

The content of this work is not subject to copyright. Design and branding are copyright @ERS 2020. This version is distributed under the terms of the Creative Commons Attribution Non-Commercial Licence 4.0. 

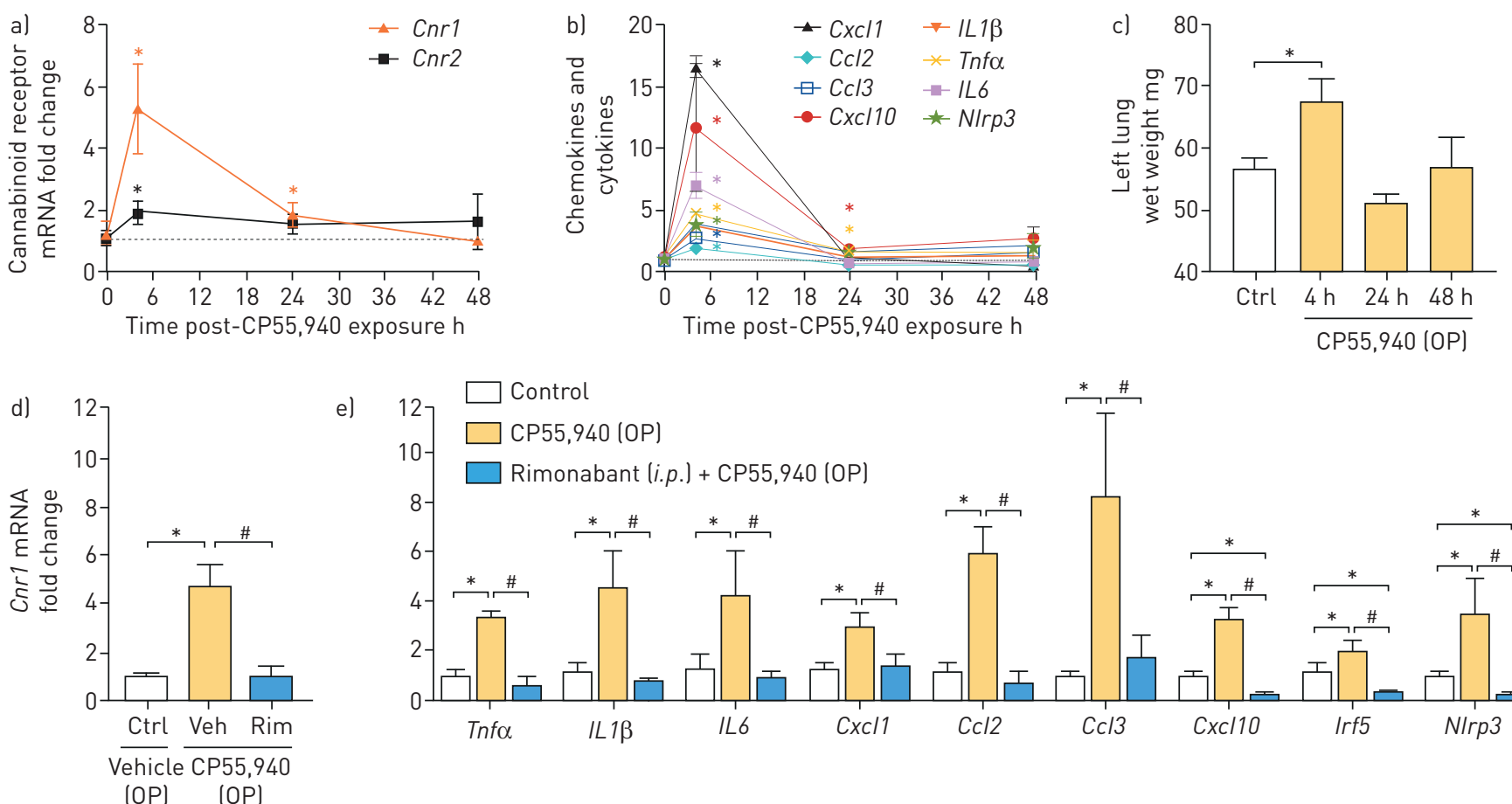

e) $\begin{array}{r}12 \\ 10-7\end{array} \begin{aligned} & \square \text { Control } \\ & \square \text { CP55,940 (OP) } \\ & \square \text { Rimonabant (i.p.) + CP55,940 (OP) }\end{aligned}$
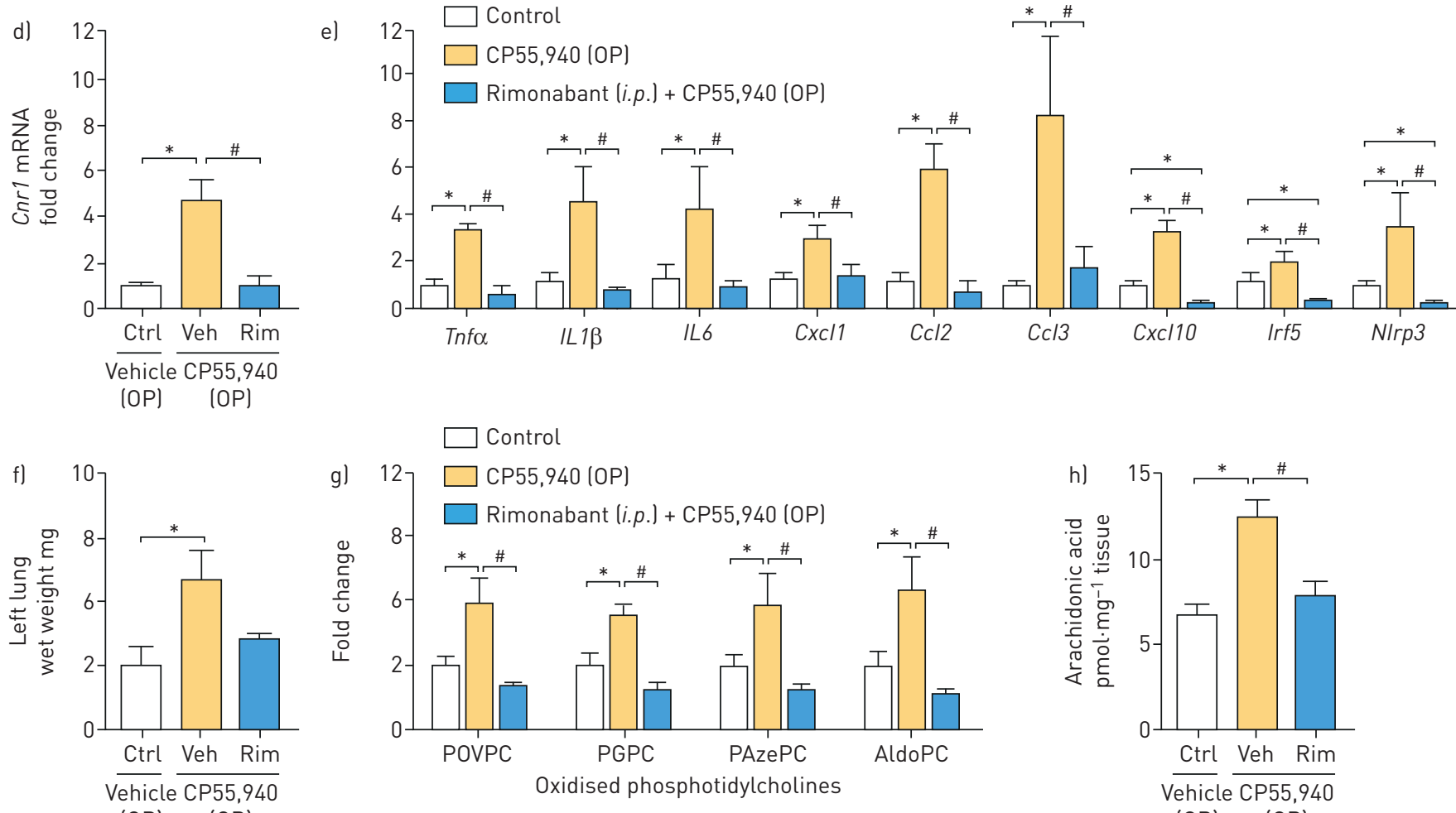

(OP) (OP)
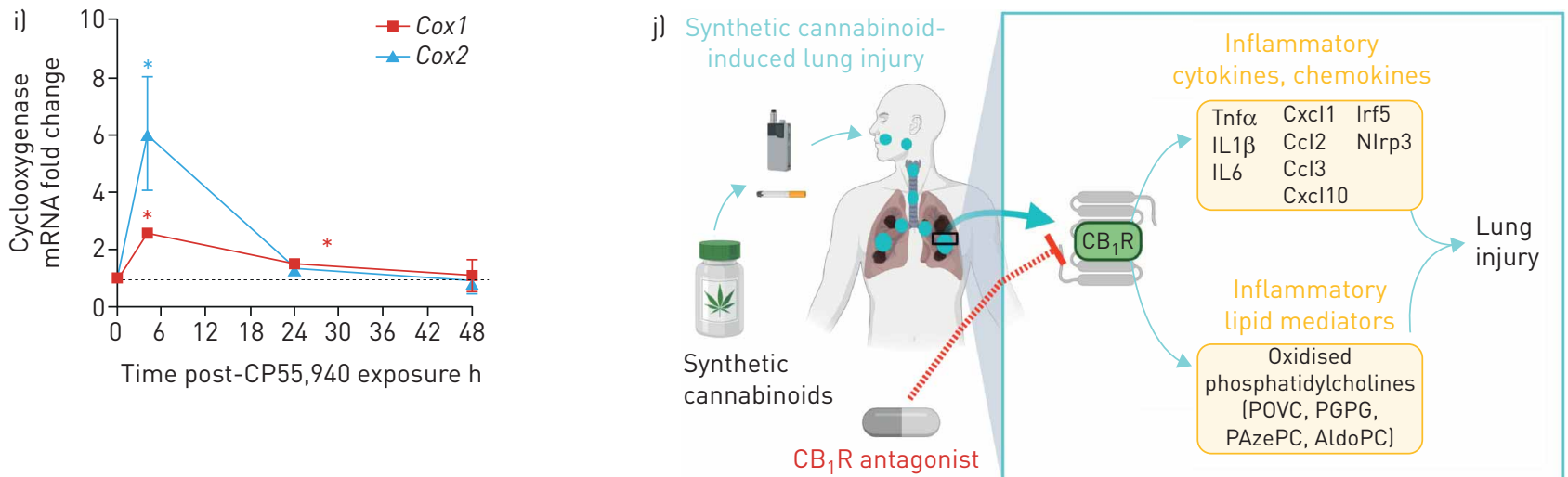

FIGURE 1 Oropharyngeal (OP) synthetic cannabinoid administration-induced gene expression of inflammatory genes and lipids via activating cannabinoid receptor $1\left(C_{1} R\right)$ in lungs. OP CP55,940 increased the gene expression of a) $C_{1} B_{1} R$ and $C B_{2} R$ receptors, and b) pro-inflammatory cytokines, chemokines and pro-inflammatory transcription factors, and $\mathrm{C}$ ) wet weight of the left lung. $\mathrm{CB}_{1} \mathrm{R}$ antagonist rimonabant (Rim) (10 $\mathrm{mg} \cdot \mathrm{kg}^{-1}$, intraperitoneal) administration prevented cannabinoid-induced gene expression of d) $C_{1} B_{1}$, and e) pro-inflammatory cytokines, chemokines and pro-inflammatory transcription factors, and f) lung wet weight; and generation of inflammatory lipids (g) oxidised phosphatidylcholines and h) arachidonic acid). i) SC induced gene expression of cyclooxygenase in lungs at 4h after OP CP55,940 administration. j) Graphical representation. The graphical representation was generated in Biorender. Data are presented as mean \pm SEM. Data were analysed by one-way ANOVA followed by Dunnett's multiple comparisons test. $n=4$ per group. POVPC: palmitoyl-oxo-valeryl-phosphotidylcholine; PGPC: palmitoyl-glutaryl-phosphotidylcholine; PAzePC: palmitoyl-azelaoyl-phosphotidylcholine; AldoPC: palmitoyl-oxo-nonanoyl-phosphotidylcholine. *: $\mathrm{p}<0.05$, versus control (Ctrl) group; ${ }^{\#}: \mathrm{p}<0.05$, versus vehicle-injected (Veh) and OP CP55,940 administered group. 
the activation of $\mathrm{CB}_{1} \mathrm{R}$ with intraperitoneal administration of the $\mathrm{CB}_{1} \mathrm{R}$ antagonist/inverse agonist rimonabant $\left(10 \mathrm{mg} \cdot \mathrm{kg}^{-1}\right) 45 \mathrm{~min}$ prior to $\mathrm{OP}$ administration of $\mathrm{CP} 55,940$, then collected tissue 4 -h post $\mathrm{CP} 55,940$. The blockade of $\mathrm{CB}_{1} \mathrm{R}$ prevented the cannabinoid-induced elevation of $\mathrm{CB}_{1} \mathrm{R}$ gene expression (figure 1d), along with inflammatory cytokines, chemokines and pro-inflammatory transcription factors (figure 1e), and lung weight (figure 1f). Rimonabant treatment reduced gene expression of Cxcl10, Irf5 and Nlrp3 below the level in the control group, possibly as a result of its inverse agonist activity that reduces constitutive activation of $\mathrm{CB}_{1} \mathrm{R}$ in lungs. These findings demonstrate that pulmonary exposure to potent cannabinoids induces lung inflammation via $\mathrm{CB}_{1} \mathrm{R}$ activation [5], which aligns with previous findings that show the pathological role of $\mathrm{CB}_{1} \mathrm{R}$ activation by endocannabinoids in alveolar inflammation $[8,9]$.

OP administration of CP55,940 also caused a significant increase in lung homogenates of palmitoyl-oxo-valeryl-phosphotidylcholine (POVPC), palmitoyl-glutaryl-phosphotidylcholine (PGPC), palmitoyl-azelaoyl-phosphotidylcholine (PAzePC) and palmitoyl-oxo-nonanoyl-phosphotidylcholine (AldoPC) in a $\mathrm{CB}_{1} \mathrm{R}$-dependent manner, as rimonabant treatment prevented such increases (figure $1 \mathrm{~g}$ ). These oxidised phosphatidylcholines (ox-PCs) are proinflammatory and instigate immune responses through binding to diverse receptors including Platelet-activating factor receptor and Toll-like receptor 4, among others [11]. Ox-PCs are implicated as lipid mediators of lung injury and acute respiratory distress syndrome (ARDS). Increases in ox-PCs like POVPC also result in their accumulation in alveolar macrophages (AM), causing foam-cell formation and M2 polarisation, both of which are associated with lung injury and fibrosis [12]. These findings suggest $\mathrm{CB}_{1} \mathrm{R}$ agonism may cause increased cytokine expression via the actions of ox-PCs, a notion evidenced previously [13]. The rapid timescale of ox-PC formation we observed suggests an enzymatic mechanism, possibly via direct action of 15-lipoxygenase (15-LO) on phospholipids, and/or indirect formation through eicosanoid incorporation into lyso-phospholipids via the Lands cycle. We observed a $\mathrm{CB}_{1} \mathrm{R}$-dependent increase in lung arachidonic acid levels (figure 1h), and a CP55,940-induced increase in pulmonary cyclooxygenases (COXs) gene expression (figure 1i), both of which support not only generation of inflammatory prostanoids, but also the possibility of indirect enzymatic ox-PC formation. The ox-PCs we report are derived from PCs which are critical components of lung surfactant. Considering their growing relevance to lung pathologies, the biologic origin of ox-PCs such as those observed in this study warrant investigation of a direct regulatory role of $\mathrm{CB}_{1} \mathrm{R}$ activation.

$\mathrm{CB}_{1} \mathrm{R}$ and $\mathrm{CB}_{2} \mathrm{R}$ have often opposite functions in pathologies. Therefore, the cannabinoid receptor system has regulatory and counter-regulatory functions. THC and many SCs have similar affinities for $\mathrm{CB}_{1} \mathrm{R}$ and $\mathrm{CB}_{2} \mathrm{R}$, the relative contribution of which to the observed effects might be context dependent considering the distinct expression levels of the two receptors in different pathologies. The findings reported in this study indicate that $\mathrm{SC}$ binding to $\mathrm{CB}_{1} \mathrm{R}$ in the lungs may instigate the pulmonary conditions observed in those hospitalised for SC use, and we posit a pathologic mechanism mediated by ox-PC signalling. Additionally, the findings suggest the potential use of $\mathrm{CB}_{1} \mathrm{R}$ antagonists in the treatment of SC-induced lung disease (figure $1 \mathrm{j}$ ). Although the $\mathrm{CB}_{1} \mathrm{R}$ antagonist rimonabant is no longer available due to neuropsychiatric side effects, orally bioavailable, peripherally restricted $C_{1} R$ antagonists which are currently under clinical development may offer an alternative [8, 14]. Recent observational studies have uncovered a unique imaging pattern distinct to SC use as detected by multidetector computed tomography, which can serve as a diagnostic criterion for SC-induced pulmonary disease. This may be of particular importance when the patient is unresponsive [6].

There is also growing concern that THC itself may also cause similar respiratory pathologies. As the popularity and availability of THC-rich cannabis concentrates increases, there are reports of ARDS associated with its vaporised inhalation [15]. Cannabis concentrate-induced ARDS presents with similar findings as SC-induced ARDS, including alveolar haemorrhage, organising pneumonia and pulmonary infiltrates [15]. Further investigation is warranted to determine whether $C_{1} R$ activation is causal in this condition and whether concentrates might elicit such pathology due to their higher THC content or to lower levels of other cannabis constituents such as cannabidiol and tetrahydrocannabivarin, which can counter-balance THC's activity at $\mathrm{CB}_{1} \mathrm{R}$. All in all, these findings should raise an important public health concern for respiratory health related to cannabinoid use.

\section{Charles N. Zawatsky ${ }^{1,2}$, Jasmina Abdalla ${ }^{1,2}$ and Resat Cinar $\oplus^{1}$}

${ }^{1}$ Laboratory of Physiologic Studies, National Institute on Alcohol Abuse and Alcoholism, National Institutes of Health, Rockville, MD, USA. ${ }^{2}$ These authors contributed equally.

Correspondence: Resat Cinar, Laboratory of Physiologic Studies, National Institute on Alcohol Abuse and Alcoholism, National Institutes of Health, 5625 Fishers Lane, Bethesda, MD 20892-9304, USA. E-mail: resat.cinar@nih.gov 
Received: 6 March 2020 | Accepted after revision: 18 June 2020

Author contributions: R. Cinar conceived and designed the study, and analysed and interpreted the data. C.N. Zawatsky and J. Abdalla acquired the data. C.N. Zawatsky and R. Cinar wrote the manuscript.

Conflict of interest: None declared.

Support statement: This study was supported by intramural research funds from the National Institute on Alcohol Abuse and Alcoholism. Funding information for this article has been deposited with the Crossref Funder Registry.

\section{References}

1 Gunderson EW, Haughey HM, Ait-Daoud N, et al. A survey of synthetic cannabinoid consumption by current cannabis users. Subst Abus 2014; 35: 184-189.

2 Cordeiro SK, Daro RC, Seung H, et al. Evolution of clinical characteristics and outcomes of synthetic cannabinoid receptor agonist exposure in the United States: analysis of National Poison Data System data from 2010 to 2015 Addiction 2018; 113: 1850-1861.

3 Martz G, Tankersley W, Mekala HM, et al. Rates of synthetic cannabinoid use in adolescents admitted to a treatment facility. Prim Care Companion CNS Disord 2018; 20: 17m02265.

4 Alon MH, Saint-Fleur MO. Synthetic cannabinoid induced acute respiratory depression: case series and literature review. Respir Med Case Rep 2017; 22: 137-141.

5 Alhadi S, Tiwari A, Vohra R, et al. High times, low sats: diffuse pulmonary infiltrates associated with chronic synthetic cannabinoid use. J Med Toxicol 2013; 9: 199-206.

6 Berkowitz EA, Henry TS, Veeraraghavan S, et al. Pulmonary effects of synthetic marijuana: chest radiography and CT findings. AJR Am J Roentgenol 2015; 204: 750-757.

7 Loschner A, Cihla A, Jalali F, et al. Diffuse alveolar hemorrhage: add "greenhouse effect" to the growing list. Chest J 2011; 140: 149A.

8 Cinar R, Gochuico BR, Iyer MR, et al. Cannabinoid CB1 receptor overactivity contributes to the pathogenesis of idiopathic pulmonary fibrosis. JCI Insight 2017; 2: e92281.

9 Bronova I, Smith B, Aydogan B, et al. Protection from radiation-induced pulmonary fibrosis by peripheral targeting of cannabinoid receptor-1. Am J Respir Cell Mol Biol 2015; 53: 555-562.

10 McGregor IS, Issakidis CN, Prior G. Aversive effects of the synthetic cannabinoid CP 55,940 in rats. Pharmaco Biochem Behav 1996; 53: 657-664.

11 Bochkov VN, Oskolkova OV, Birukov KG, et al. Generation and biological activities of oxidized phospholipids. Antioxid Redox Signal 2010; 12: 1009-1059.

12 Romero F, Shah D, Duong M, et al. A pneumocyte-macrophage paracrine lipid axis drives the lung toward fibrosis. Am J Respir Cell Mol Biol 2015; 53: 74-86.

13 Subbanagounder G, Wong JW, Lee H, et al. Epoxyisoprostane and epoxycyclopentenone phospholipids regulate monocyte chemotactic protein-1 and interleukin-8 synthesis. Formation of these oxidized phospholipids in response to interleukin-1beta. J Biol Chem 2002; 277: 7271-7281.

14 Cinar R, Iyer MR, Kunos G. The therapeutic potential of second and third generation CB1R antagonists. Pharmacol Ther 2020; 208: 107477.

15 He T, Oks M, Esposito M, et al. 'Tree-in-bloom': severe acute lung injury induced by vaping cannabis oil. Ann Am Thorac Soc 2017; 14: 468-470. 